\title{
VARIATION OF SPECTRAL CHARACTERISTICS OF COELENTERAMIDE-CONTAINING FLUORESCENT PROTEIN FROM OBELIA LONGISSIMA EXPOSED TO DIMETHYL SULFOXIDE
}

\author{
A. S. Petrova, ${ }^{1,3}$ R. R. Alieva, ${ }^{1}$ N. V. Belogurova,${ }^{1}$ \\ L. S. Tirranen ${ }^{1,3}$, and N. S. Kudryasheva ${ }^{1,2}$
}

UDC 577.345, 577.34

Effect of dimethyl sulfoxide (DMSO), a widespread biomedical agent, on spectral-luminescent characteristics of coelenteramide-containing fluorescent protein - discharged obelin - is investigated. Contributions of violet and blue-green spectral components to fluorescence of discharged obelin are elucidated and characterized at different photoexcitation energies. Dependences of these contributions on the DMSO concentration are presented. Spectral changes are related to the destructive effect of DMSO on fluorescent protein and decreasing efficiency of proton transfer to electronically excited states of fluorophore.

Keywords: fluorescent coelenteramide-containing fluorescent proteins, discharged obelin, proton transfer, dimethyl sulfoxide.

\section{INTRODUCTION}

Fluorescent proteins are modern and very promising tools for biological and medical investigations. These proteins are synthesized within cells and can be used as biological fluorescent markers for monitoring of intracellular processes. Fluorescent protein is polypeptide comprising aromatic fluorophore. The most famous fluorescent protein is green fluorescent protein (GFP) [1, 2]. In addition, coelenteramide-containing proteins also belong to the group of fluorescent proteins. Their fluorophore is the coelenteramide molecule (Fig. 1) non-covalently bonded with protein inside its hydrophobic cavity. Unlike GFPs, coelenteramide-containing fluorescent proteins are not so widespread in biomedical investigations and their potential as color biomarkers is currently underestimated.

Fluorophore of discharged photoproteins - coelenteramide - is chemically active in excited state and capable of producing a recoil proton with the formation of various fluorescent forms [3] that differ by the energy of fluorescent states and hence fluorescence color. Figure 1 shows schematically the energy level diagram of the excited states of protonated and partially protonated coelenteramide forms that are responsible for violet and blue-green components of the fluorescence spectra of coelenteramide and coelenteramide-containing proteins. The energy of the state $S_{1}{ }^{* \prime}$ is determined by the effective position of the proton of the coelenteramide phenolic group with respect to the protonacceptor fragment of the histidine amino acid residue (His22) (Fig. 1) in the protein surrounding. Wherein the relationship between the violet and blue-green components is determined by the deprotonation of the coelenteramide phenolic group in the excited state $S_{1}$ * that, in its turn, is determined by the microsurrounding of coelenteramide in proteins or solvents [4-10].

${ }^{1}$ Institute of Biophysics of the Siberian Branch of the Russian Academy of Sciences, Krasnoyarsk, Russia, e-mail: aileenpetpova@gmail.com; alieva_rosa@mail.ru; nbelogurova@mail.ru; ${ }^{2}$ Siberian Federal University, Krasnoyarsk, Russia, e-mail: kudr@ibp.ru; ${ }^{3}$ Krasnoyarsk State Agrarian University, Krasnoyarsk, Russia, e-mail: 1-tiran@ya.ru. Translated from Izvestiya Vysshikh Uchebnykh Zavedenii, Fizika, No. 4, pp. 87-92, April, 2016. Original article submitted February 16, 2016. 


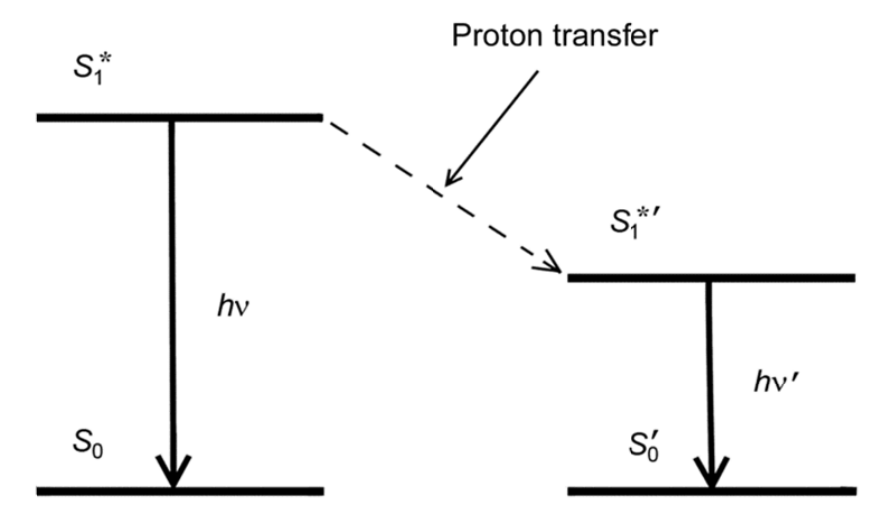

Protonated form

Partially protonated form<smiles>C[14CH2]c1ccc(CC(=O)Nc2ncc(-c3ccc(O)cc3)nc2Cc2ccccc2)cc1</smiles>

Fig. 1. Chemistry in the electronically excited state of coelenteramide - fluorophore of fluorescent coelenteramide-containing proteins.

Of particular current interest is coelenteramide-containing fluorescent protein extracted from the hydroid polyp Obelia longissima usually called discharged obelin. It is produced and studied at the Institute of Biophysics of the Siberian Branch of the Russian Academy of Sciences (IBP SB RAS, Krasnoyarsk, Russia) [10-22]. Fluorescent properties of discharged obelin are investigated theoretically [4-10] and experimentally [20,21]. Spectral components of complex fluorescent spectra of discharged obelin are investigated including time-resolved experiments [23]; they are assigned to different coelenteramide fluorescent forms [24]. Changes in the spectral composition of the fluorescence are induced by the change of calcium concentration [25], chronic effects of high temperature [26] and alcohols [27], as well as variation of photoexcitation energy [26,27]. Since obelin has a wide spectrum of potential applications in biomedical investigations, it seems important to investigate the effect of accompanying compounds on its luminescent properties. One of such compounds is dimethyl sulfoxide (DMSO) widely used in biomedical investigations, in particular, as a cryoprotectant [28].

In the present work, the effect of DMSO on the spectral-luminescent characteristics of coelenteramidecontaining fluorescent protein - discharged obelin - is investigated. Contributions of the violet and blue-green spectral components to the fluorescence of discharged obelin are elucidated and characterized at different photoexcitation energies. Dependences of these contributions on the DMSO concentrations are demonstrated. Changes in the fluorescent protein spectra are related to the efficiency of the proton transfer in the excited state of its fluorophore coelenteramide.

\section{MATERIALS AND METHODS OF RESEARCH}

Recombination preparation of obelin extracted from the hydroid polyp Obelia longissima was produced in the Laboratory of Photobiology of the IBP SB RAS [13, 14]. Discharged obelin was produced by addition of calcium chloride to an obelin solution. The total calcium chloride concentration was $0.0047 \mathrm{M}$. The reaction was conducted in 
a $0.02 \mathrm{M}$ Tris-HCL buffer solution $(\mathrm{pH}=7.0)$ and $0.005 \mathrm{M}$ EDTA. The obelin concentration in the examined solution was $10^{-5} \mathrm{M}$. DMSO was added to the obelin solution after the bioluminescent reaction immediately before the registration of the spectra. The DMSO concentration was varied from 0.002 to $2.65 \mathrm{M}$ (from 0.016 to 18.9 vol.\%). The fluorescent spectra were registered with the use of PerkinElmer LS55 fluorescence spectrometer (USA). The temperature of the cell was $20^{\circ} \mathrm{C}$. The parameters of registration of the spectra were the following: the wavelength range was $300-750 \mathrm{~nm}$ and the photoexcitation wavelengths were 280 and $350 \mathrm{~nm}$.

The complex fluorescence spectra of discharged obelin were deconvolved into components using Gaussian distribution. Mathematical processing was performed with the software packages Origin 8.5.1 and Matlab 8.0. To determine the number and position of maxima of the spectral components, the second derivative method was used. The spectra were deconvolved into individual Gaussian components in the coordinates: fluorescence intensitywavelength $[28,29]$. The deviation $d$ of the calculated spectrum from the experimental one was evaluated as follows:

$$
d=\frac{\left|S_{\exp }-\sum S_{\text {comp }}\right|}{S_{\exp }} \cdot 100 \%,
$$

where $S_{\text {exp }}$ is the area of the experimental spectrum and $S_{\text {comp }}$ is the area of the individual spectral component. The value of $d$ did not exceed $0.5 \%$.

In the short-wavelength range ( $<380$ and $<300 \mathrm{~nm}$ for photoexcitatioin wavelengths of 350 and $280 \mathrm{~nm}$, respectively), the spectra were complemented using the Gaussian distribution. Contribution $W$ of the spectral components to the overall photoluminescence spectrum was calculated using the following formula:

$$
W=\frac{S_{\text {comp }}}{\sum S_{\text {comp }}} .
$$

The effect of DMSO on the fluorescence intensity of discharged obelin was estimated using the empirical fluorescence quenching constant $K$ :

$$
I / I_{0}=e^{-K C}
$$

where $I$ and $I_{0}$ are the fluorescence intensities in the presence and absence of DMSO, respectively, and $C$ is the DMSO concentration, in M.

\section{RESULTS AND DISCUSSION}

Fluorescent characteristics of discharged obelin in DMSO solutions were investigated. Figure 2 shows the normalized fluorescence spectra of discharged obelin at photoexcitation wavelengths of 280 and $350 \mathrm{~nm}$, corresponding to its absorption maxima. It can be seen that the obelin fluorescence is characterized by the maximum at $503 \mathrm{~nm}$ upon photoexcitation by $350 \mathrm{~nm}$ and includes an additional band in the near-ultraviolet range with the maximum at $346 \mathrm{~nm}$ upon photoexcitation by $280 \mathrm{~nm}$.

Figure 3 shows dependences of the fluorescence intensities of discharged obelin on the DMSO concentration at two photoexcitation energies -280 and $350 \mathrm{~nm}$. It can be seen that increasing DMSO concentration leads to decreasing maximum intensity in the visible spectral range in both cases $(503 \mathrm{~nm}$, curves 1 and 3$)$. Wherein the intensity of the ultraviolet maximum (346 nm, curve 2) increases with DMSO concentration.

The fluorescence quenching constants $K$ were calculated from Eq. (3) using three dependences shown in Fig. 3. Values of these constants differed by approximately two orders of magnitude in the intervals of low and high DMSO concentrations. For example, the $K$ values for curve 3 (Fig. 3) were equal to 42 and $0.32 \mathrm{M}^{-1}$, respectively. Such differences in the constants can testify to different mechanisms of fluorescence quenching at low and high DMSO concentrations related, probably, to the protein destruction. 

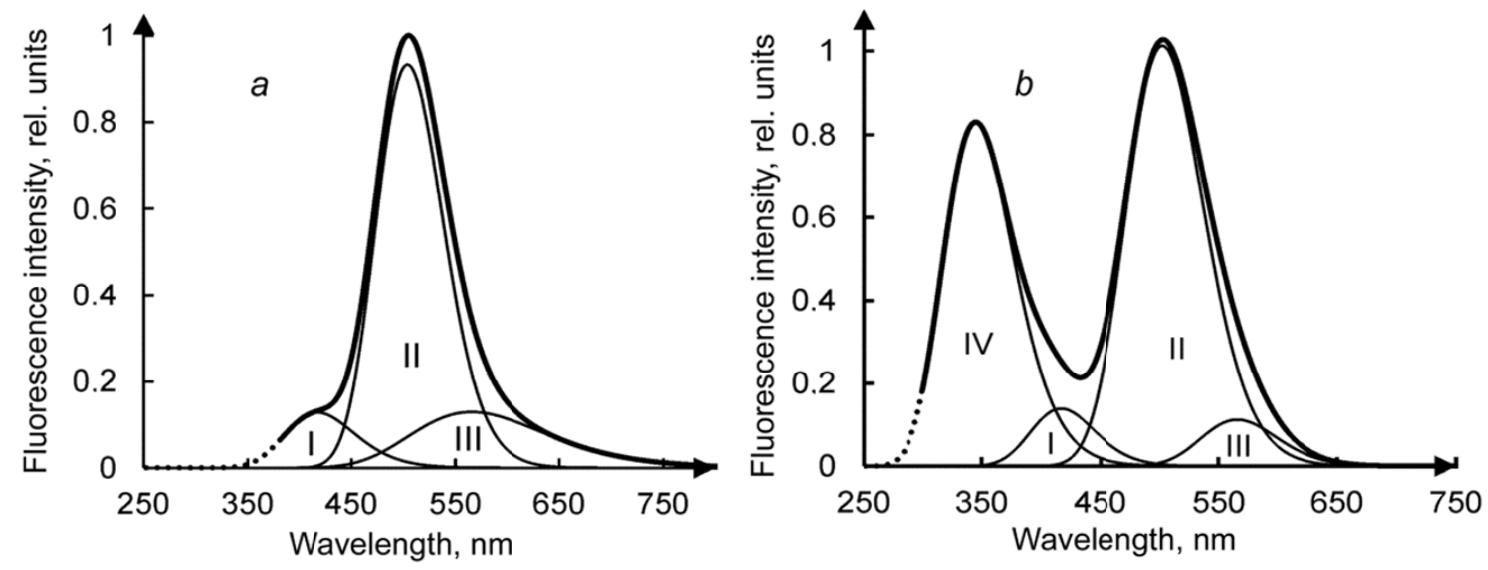

Fig. 2. Fluorescence spectra of discharged obelin (bold curves) and their components $(\mathrm{I}-\mathrm{IV})$ at $C_{\mathrm{DMSO}}=0.4 \mathrm{M}: \lambda_{\mathrm{exc}}=350(a)$ and $280 \mathrm{~nm}(b)$.

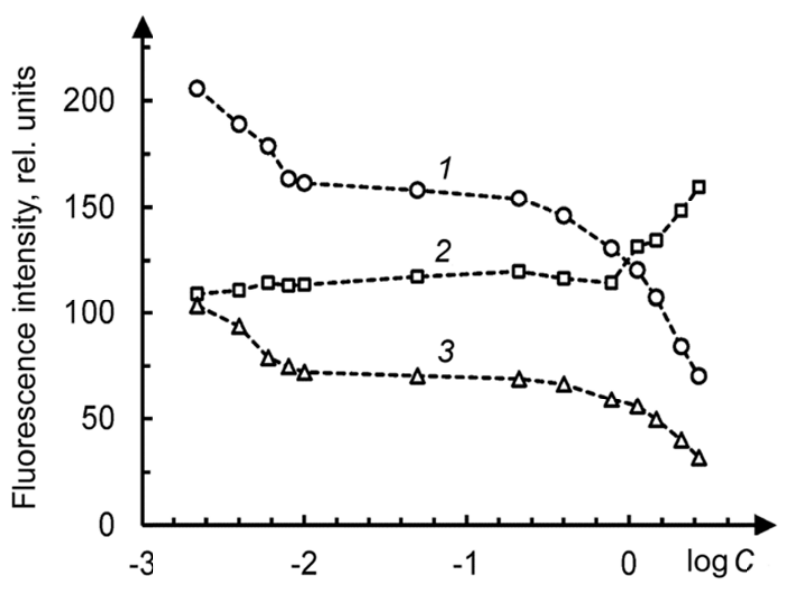

Fig. 3. Dependence of the intensity of the discharged obelin fluorescence on the DMSO concentration $C$, in M. Here curve 1 is for $\lambda_{\max }=503 \mathrm{~nm}\left(\lambda_{\text {exc }}=280 \mathrm{~nm}\right)$, curve 2 is for $\lambda_{\max }=346 \mathrm{~nm}\left(\lambda_{\mathrm{exc}}=280 \mathrm{~nm}\right)$, and curve 3 is for $\lambda_{\max }=503 \mathrm{~nm}\left(\lambda_{\mathrm{exc}}=350 \mathrm{~nm}\right)$.

All obtained spectra were deconvolved into Gauss components. Figure 2, as an example, shows the fluorescence spectra of discharged obelin and their components at $C_{\mathrm{DMSO}}=0.4 \mathrm{M}$. It can be seen that the emission spectrum at $350 \mathrm{~nm}$ photoexcitation is the superposition of three Gaussian components in the visible spectral range with maxima at 420, 503, and $565( \pm 1) \mathrm{nm}$ (components I, II, and III, respectively, in Fig. 2a). The emission spectrum at $280 \mathrm{~nm}$ photoexcitation includes additional component IV with maximum at $345 \mathrm{~nm}$ in the ultraviolet spectral range (Fig. $2 b$ ). It should be noted that variation in DMSO concentration had no effect on the wavelength of maxima for components I-IV, but caused a change in their contributions.

The components were assigned to different fluorescent forms of coelenteramide on the basis of the literature data $[3,30,31]$. Component I was assigned to the protonated coelenteramide form (see Fig. 1) [30, 32, 33]. Fluorescence emission of the amide anion was not detected, which is in agreement with the data of Hirano et al. [4, 32]. Component II was assigned to the complex with partial proton transfer (Fig. 1) to the proton-acceptor group His2 of amino acidic coelenteramide surrounding, whereas component III with maximum at $565 \mathrm{~nm}$ was assigned to the 

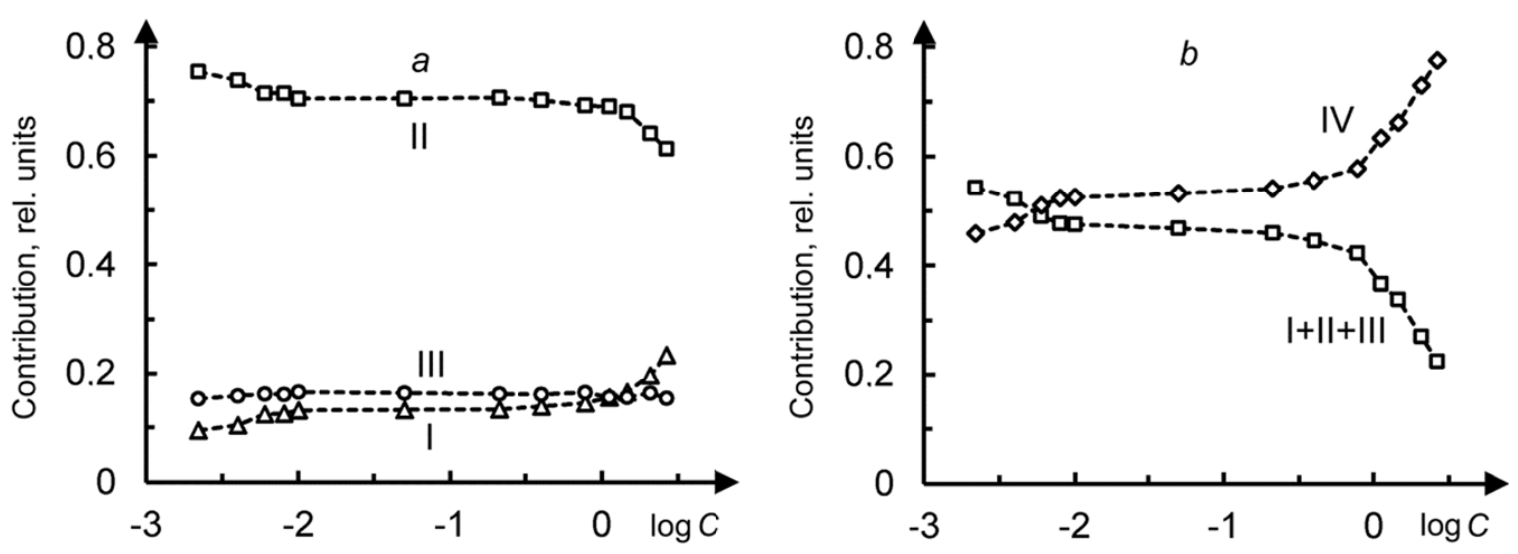

Fig. 4. Contributions of components I, II, III, and IV to the emission spectra of discharged obelin versus the DMSO concentrations. Here $\lambda_{\mathrm{exc}}=350(a)$ and $280 \mathrm{~nm}(b)$.

pyrazine anion (that is, the resonance structure of the phenolate anion). Component IV corresponds to isolated fragments with a low degree of conjugation in the protein-coelenteramide complex. It can include fluorescence contributions of aromatic fragments of amino acid residues of protein as well as fragments of the coelenteramide molecule.

The contributions of the individual components to the fluorescence spectrum of discharged obelin are calculated for different DMSO concentrations. Figure $4 a$ shows contributions of components I-III to the visible fluorescence. It can be seen that an increase of the DMSO concentration causes the contribution of protonated violet component I to increase, the contribution of the complex with partial proton transfer (blue-green component II) to decrease, and the contribution of protonated violet component I to increase almost threefold when the DMSO concentration increases to its maximum $(2.65 \mathrm{M})$.

The growth of the contribution of the protonated coelenteramide form (violet component) with increasing DMSO concentration indicates a decrease in the probability of proton transfer in the excited coelenteramide state in protein, which probably is caused by the increase in the distance between the phenol coelenteramide group and His22 (Fig. 1) due to the partial protein denaturation.

An increase in the contributions of ultraviolet component IV with increasing DMSO concentration can be seen from Fig. $4 b$. This result testifies also to the structural change of coelenteramide-containing fluorescent protein caused by the DMSO effect.

\section{CONCLUSIONS}

Discharged obelin is a representative of the group of coelenteramide-containing fluorescent proteins. Due to its stability and non-toxicity, it is a promising tool for application as a fluorescent marker for visualization of biochemical processes in various biomedical investigations. As shown by previous studies, the fluorescence spectra of discharged obelin are subjected to changes under the effect of various physicochemical factors [25-27]. These changes depend on microsurrounding of coelenteramide in protein and are determined by the effective position of the proton of the phenol coelenteramide group in the excited state $S_{1}^{*}$ (Fig. 1). This factor determines the color characteristics of fluorescence of discharged obelin.

In the present work, we have established that the intensity and shape of the fluorescence spectra of discharged obelin depend on the DMSO concentration. The fluorescence intensity in the visible spectral range increases, and the intensity of the ultraviolet spectral component increases with rising DMSO concentration. The increase in the contribution of the violet component and the decrease in the contribution of the blue-green spectral component are caused by the decrease in the efficiency of proton transfer in the excited coelenteramide state upon exposure to DMSO. 
The efficiency of the process decreases due to the change of interatomic distances in amino acid coelenteramide surrounding in protein under the destructive effect of DMSO. The increase in the contribution of the ultraviolet component also testifies to the structural change of coelenteramide-containing fluorescent protein exposed to DMSO. The contributions of various groups of this protein to the ultraviolet luminescence will be elucidated in subsequent investigations including time-resolved experiments.

This work was supported in part by the Russian Science Foundation (Contract No. 14-14-00076).

\section{REFERENCES}

1. O. V. Stepanenko, V. V. Verkhusha, I. M. Kuznetsova, et al., Tsitologiya, 49, No. 5, 395-420 (2007).

2. $\quad$ N. N. Zubova, A. Yu. Bulavina, and A. P. Savitskii, Usp. Biol. Khim., 43, 163-224 (2003).

3. O. Shimomura and K. Teranishi, Luminescence, 15, 51-58 (2000).

4. $\quad$ C. Min, Z.-Ch. Li, A.-M. Ren, et al., J. Photochem. Photobiol., A251, 182-188 (2013).

5. S. F. Chen, N. Ferre, and Y. J. Liu, Chem. Eur. J., 19, 8466-8472 (2013).

6. S. F. Chen, I. Navizet, D. Roca-Sanjua, et al., J. Chem. Theory Comp., 8, 2796-2807 (2012).

7. O. N. Chaikovskaya, E. A. Karetnikova, I. V. Sokolova, et al., Russ. Phys. J., 51, No. 12, 1344-1355 (2008).

8. Z.-S. Li, L.-Y. Zou, Ch.-G. Min, et al., J. Photochem. Photobiol., B127, 94-99 (2013).

9. Z.-S. Li, X. Zhao, L.-Y. Zou, et al., Photochem. Photobiol., 89, 849- 855 (2013).

10. L. Yu. Antipina and S. G. Ovchinnikov, V Mire Nauch. Otkr. Fiz.-Mat. Nauki, 5, No. 11, 35-36 (2010).

11. E. S. Vysotskii, S. V. Markova, and L. A. Frank, Mol. Biol., 40, No. 3, 355-367 (2006).

12. E. S. Vysotskii, V. S. Bondar', and B. N. Letunov, Biokhimiya, 54, 965-973 (1989).

13. B. A. Illarionov, L. A. Frank, V. A. Illarionova, et al., Meth. Enzymol., 277, 223-249 (2000).

14. S. V. Markova, E. S. Vysotski, J. R. Blinks, et al., Biochemistry, 41, 2227-2236 (2002).

15. E. S. Vysotski and J. Lee, Accounts Chem. Res., 37, 405-415 (2004).

16. P. V. Natashin, S. V. Markova, J. Lee, et al., FEBS J., 281, 1432-1445 (2014).

17. E. V. Eremeeva, P. V. Natashin, L. Song, et al., ChemBioChem, 14, 739-745 (2013)

18. P. V. Natashin, W. Ding, E. V. Eremeeva, et al., Acta Crystallograph., D70, 720-732 (2014).

19. E. V. Eremeeva, S. V. Markova, A. H. Westphal, et al., FEBS Lett., 583, 1939-1944 (2009).

20. E. V. Eremeeva, S. V. Markova, L. A. Frank, et al., Photochem. Photobiol. Sci., 12, 1016-1024 (2013).

21. E. V. Eremeeva, S. V. Markova, W. J. H. Berkel, and E. S. Vysotski, J. Photochem. Photobiol., B127, 133139 (2013).

22. N. V. Belogurova, N. S. Kudryasheva, and R. R. Alieva, J. Mol. Struct., 924-926, 148-152 (2009).

23. B. Van Oort, E. V. Eremeeva, R. B. M. Koehorst, et al., Biochemistry, 48, 10486-10491 (2009).

24. N. V. Belogurova, N. S. Kudryasheva, R. R. Alieva, et al., J. Photochem. Photobiol., B92, No. 2, 117122 (2008).

25. N. Belogurova and N. Kudryasheva, J. Photochem. Photobiol., B101, 103-108 (2010).

26. R. R. Alieva, N. V. Belogurova, A. S. Petrova, et al., Analyt. Bioanalyt. Chem., 405, No. 10, 33513358 (2013).

27. R. R. Alieva, N. V. Belogurova, A. S. Petrova, et al., Analyt. Bioanalyt. Chem., 406, No. 12, 29652974 (2014).

28. K. B. Yatsimirskii and T. V. Mel'kova, Spectroscopic Methods in the Chemistry of Complex Compounds [in Russian], Moscow (1984).

29. V. I. Mikhailenko and Yu. R. Red'kin, J. Appl. Spectr., 31, No. 5, 1444-1445 (1979).

30. K. Mori, S. Maki, H. Niwa, et al., Tetrahedron, 62, 6272-6288 (2006).

31. Y. Imai, T. Shibata, S. Maki, et al., J. Photochem. Photobiol., A146, 95-107 (2001).

32. T. Hirano, Y. Ohmiya, S. Maki, et al., Tetrahedron Lett., 39, 5541-5544 (1998).

33. E. S. Vysotski, Z. J. Liu, S. V. Markova, et al., Biochemistry, 42, 6013-6024 (2003). 